\title{
Foot-placement accuracy during planned and reactive target stepping during walking in stroke survivors and healthy adults.
}

\author{
Susanne M. van der Veen ${ }^{1}$, Ulrike Hammerbeck ${ }^{2}$, Kristen L. Hollands ${ }^{3}$. \\ 1 Virginia Commonwealth University, Department of Physical Therapy, Richmond VA, USA. \\ Email: smvanderveen@vcu.edu \\ 2 Manchester University, Physical Therapy, Manchester, UK \\ 3 Salford University, Health science, Salford, UK
}

\begin{abstract}
Background: The high prevalence of falls due to trips and slips following stroke may signify difficulty adjusting foot-placement in response to the environment. However, little is known about under what circumstances footplacement adjustment becomes difficult for stroke survivors (SS), making the design of targeted rehabilitation interventions to improve independent community mobility difficult.
\end{abstract}

Research question: To investigate the effect of planned and reactive target-stepping on foot-placement accuracy in stroke survivors and young and older healthy adults?

Methods: Young ( $\mathrm{N}=11,30 \pm 6$ years) and older $(\mathrm{N}=10,64 \pm 8$ years) healthy adults and $\mathrm{SS}(\mathrm{N}=11,67 \pm 9$ years) walked, at preferred pace, on a force instrumented treadmill. Each participant walked to illuminated targets, visible two steps in advance (planned) or appearing at contralateral midstance (reactive). Foot-placement error (magnitude and bias) and number of missed targets were compared.

Results: All participants missed more reactive than planned targets $(\mathrm{p}=0.05)$, and SS missed more targets than young $(\mathrm{p}<0.001)$ and older $(\mathrm{p}=0.001)$ adults. But no interaction showing SS missed more reactive targets than other groups was found. For all groups: reactive adaptations to steps in the anterio-posterior plane resulted in lower error than planned adaptations $(\mathrm{p}=0.027)$. Lengthening steps where undershot more than shortening $(\mathrm{p}<0.001)$ by all groups. Reactive medio-lateral adaptations over all induced larger error $(\mathrm{p}=0.029)$ than planned and changed the direction of bias $(\mathrm{p}=0.018)$.

Significance: SS experience difficulty making all adjustments, they showed increased error in all conditions but less pronounced difference between planned and reactive stepping. SS may use a reactive control strategy for all adjustments, in contrast to healthy young adults who may plan foot-placement in advance. The likelihood of stroke survivors misplacing a step is large, with $9.8 \%$ targets missed; possibly leading to falls. Further investigation is needed to understand foot-placement control strategies used by SS and the role of planning in gait adaptability. 


\section{Introduction}

It is essential for independent mobility to be able to adapt walking to avoid obstacles and target safe, level, footfall locations in cluttered environments. While many stroke survivors (SS) regain some mobility, between 43-70\% of SS experience falls [1-5], mostly due to trips, slips, and misplaced steps while walking [6, 7]. It is likely that gait adaptations to the environment is a fall risk factor in independently mobile SS. Recent studies demonstrated the ability to identify prospective fallers[8] due to the fact that accurate foot-placement control is correlated with balance and walking-adaptability [9]However, little is known about how and under what circumstances footplacement adjustment becomes difficult for SS, making the design of targeted rehabilitation interventions to improve independent community mobility difficult.

The time available to plan and execute foot-placement adjustment is important. In uncluttered terrain, walking over clear level paths, young healthy adults (YH) look about two steps ahead. In more cluttered terrain, attention is shifted closer [10,11], affording less time to plan and adjust steps to navigate terrain. In $\mathrm{YH}$, this reduced planning time does not compromise foot-placement up until late swing [10, 12]. However, YH and OH slow their walking speed when adjusting foot-placement to visual cues [13]. Collectively, these observations indicate that while it is possible to execute adjustments to foot-placement in a very short time frame, YH prefer to afford themselves time to plan steps either by looking ahead and/or slowing down.

Investigations of foot-placement adjustments in SS have only been carried out in stepping from standing [14] or avoiding obstacles $[15,16]$. Decreased response time elicits a step lengthening strategy more often when avoiding obstacles [15], in addition to SS being less successful avoiding obstacles[15, 16]. This lengthening strategy possibly coincides naturally with prolonging the ongoing avoidance step, allowing for more adjustment time [17]. Whereas shortening a step requires a termination of an ongoing step. Terminating a step may be challenging following stroke due to delayed muscle activation seen when adjusting foot-placement to targets [14] and avoiding obstacles [18].

Improved understanding of foot-placement adaptability may aid development of rehabilitation interventions for safe independent mobility in SS. We hypothesize that foot-placement accuracy declines when foot-placement adjustments are made reactively, affecting SS more severely than HO and YH counterparts. Additionally, step adjustments which narrow/shorten the base of support are expected to increase foot-placement error more than widening/lengthening steps. Therefore, the aims of this study are to address the following questions: 1) How is the accuracy of foot-placement affected when adjusting steps reactively for SS and YH and older adults (OH)? 2) Are there differences in foot-placement accuracy between the paretic and non-paretic leg and according to direction of step adjustment when adjusting steps reactively? 


\section{Methods}

\section{Participants}

We recruited $\mathrm{YH}$ (18-40 years old), $\mathrm{OH}$ (age matched \pm 5 years with SS individual) and SS to participate in the study [19]. For SS, inclusion criteria were: $>6$ months post-stroke, able to walk $10 \mathrm{~m}$ independently without orthopedic aids or assistance <30s. Exclusion criteria for both healthy participants and SS included neuromusculoskeletal (apart from stroke) conditions affecting walking ability and receptive and/or language problems that could preclude informed consent. In line with research governance policies in the UK, the University ethics committee (HSR1617-27) approved the study and all participants provided written informed consent prior to participation in line with the declaration of Helsinki.

Demographic and anthropometric data collected included as shown in Table 1.

\section{Apparatus}

A treadmill with a single embedded force platform (C-Mill, MotekForcelink, Culemborg, The Netherlands) was used allowing for online gait event detection while projecting visual stepping targets, as previously validated [20, 21], showing center of pressure gait event detection detected midstance with enough precision to be within the single stance window[20]. As gait speed is variable for the different groups, available response time may have been different, but adaptation of gait has shown to be linked to the gait cycle[22, 23]. A six-camera system (Qualisys, Gottenborg, Sweden) was used to track markers on the foot synchronously with measurements obtained through the software of the treadmill.

\section{Experimental setup and protocol}

Participants familiarized themselves to walking on the treadmill for roughly 3 minutes. Firstly, self-selected walking speed (SSWS) was determined by gradually increasing speed from $1 \mathrm{~km} / \mathrm{h}$ by $0.1 \mathrm{~km} / \mathrm{h}$ increments until participants reached comfortable walking speed, followed by a one-minute walking period. The second minute, targets were projected on the treadmill to allow participants to become acquainted with target stepping (CueForce1, MotekForcelink), and adjust speed if SSWS could not be maintained during preferred target stepping. In the last minute participants walked without targets at their SSWS updated for target walking speed when needed. The last 30 seconds of the familiarization a participants' step length and width were calculated to inform future target locations (custom Matlab program based on step lengths and widths recorded by CueForece1).

After the familiarization, participants completed ten trials of 100 steps each: one stepping trial with targets placed at preferred foot landings, and three trials of each of the following conditions, 1.) No targets; baseline trial, 2.) Planned targets (projected 2 steps ahead, travelling at belt speed), 3.) Reactive targets (projected at contralateral midstance or midswing of targeting leg: allowing the second half of the swing phase to adjust foot placement). In these adaptability trials, 24 targets were placed to alter preferred foot-placement ( 6 each of shortening, lengthening, 
narrowing and widening) interspersed semi-randomly with 76 preferred foot landing targets (Figure 1). SS wore a safety harness to prevent a fall, this harness did not provide any support for weight or balance.

\section{Measure of stepping performance}

Stepping error was used to measure accuracy of foot-placement control. First, the center of the foot $(\mathrm{CoF})$ was calculated from four foot markers (calcaneus, $1^{\text {st, }}$ and $5^{\text {th }}$ metatarsal head and $2^{\text {nd }}$ distal phalanx head) at midstance. Stepping error was then defined as the distance of the CoF to the centre of target. The error of foot-placement was analyzed separately in the medio-lateral (ML) and antero-posterior (AP) directions for all steps. Absolute error was used to compare the magnitude of error, and the average bias (calculated as the average of the signed error magnitude) of foot-placement was used to analyze the direction of error (undershoot vs. overshoot) between the different steps.

The size of the foot was projected around the CoF and overlaid on the target (custom Matlab 2016a). When no part of the foot was in contact with the target, this was considered a missed step. The percentage of missed steps was calculated as the total number of missed steps (including both preferred and adaptation steps) divided by the total number of steps taken in that condition ( $300=3$ trials of 100 steps per condition).

\section{Statistics}

In total, four analysis of covariance (ANCOVA)were carried out individually on each outcome measure; absolute AP error, AP bias, absolute ML error, and ML bias. The same model was used in each of these four ANCOVAs with 3 within subject factors: 1) condition (planned and reactive steps) 2) steps (preferred, widening and narrowing (ML), or preferred, shortening and lengthening (AP) steps) and 3) side (left right for healthy and paretic and non-paretic for stroke) and the between subjects factor of groups (YH, OH and SS). SSWS is known to be related to balance control and accuracy of foot-placement adjustments [9] and so was used as a covariate. Post-hoc comparisons were assessed using Bonferroni test with adjustment for multiple comparisons. A $\mathrm{p}<0.05$ was used for statistical significance. The percentage of missed targets was angular transformed to stabilize the variance and reach normal distribution [16, 24], and compared between different conditions (planned and reactive steps) with a repeated measures ANOVA.

\section{Results}

\section{Participants}

Participants (11 YH, $10 \mathrm{OH}$, and 11 SS, for demographics see Table 1) took part in the study. Treadmill walking speed was assessed according to suggested thresholds for SS [25], two participants were non-functional walkers $(<0.4 \mathrm{~m} / \mathrm{s})$, seven limited outdoor walkers $(0.4-0.8 \mathrm{~m} / \mathrm{s})$ and two healthy walkers $(>0.8 \mathrm{~m} / \mathrm{s})$. Two OH and one YH were limited outdoor walkers $(0.4-0.8 \mathrm{~m} / \mathrm{s})$ and the rest of the healthy adults walked at SSWS exceeding the $0.8 \mathrm{~m} / \mathrm{s}$ limit. It is important to remember that these thresholds are based on over ground walking, but here asses 
treadmill walking. Treadmill walking speeds are slower than over ground walking speeds, likely due to the increased metabolic cost [26] and the fact treadmill walking was set on target stepping speed therefore, these functionality assessments may underestimate participants' walking abilities. According to the suggested thresholds for Berg balance scores, one stroke survivor should be walking with an assistive device (score <40), two had higher risk of falls (score <45)[27]. Based on the TUG six SS would be at increased risk of falls [28] and three of these six also have an increased falls risk indicated by the 10-item dynamic gait index [29].

\section{Step lengthening and shortening}

The absolute (or magnitude) error in AP direction was not significantly different between conditions (reactive $4.7 \pm 0.5 \mathrm{~cm}$ planned $5.3 \pm 0.6 \mathrm{~cm}$ ) (Figure $2 \mathrm{~A}$ ). However, an interaction effect of step direction and group was found $\left(\mathrm{F}(4,56)=2.910, \mathrm{p}=0.029, \eta \mathrm{p}^{2}=0.172\right)$, where $\mathrm{YH}$ had less error in lengthening $(3.0 \pm 1.2 \mathrm{~cm})$ than shortening $(5.3 \pm 0.7 \mathrm{~cm})$ steps, $\mathrm{OH}$ and $\mathrm{SS}$ had larger errors lengthening $(5.7 \pm 1.2 \mathrm{~cm}, 8.3 \pm 1.2 \mathrm{~cm}$, respectively) than shortening $(3.5 \pm 0.7 \mathrm{~cm}, 4.8 \pm 0.7 \mathrm{~cm}$, respectively) their step. Overall, absolute error was greater for lengthening steps than shortening steps $(\mathrm{F}(2,27)=4.548, \mathrm{p}=0.002, \eta \mathrm{p} 2=0.252)$.

When investigating the bias (or direction of error), no main effect of group or response time was found in AP direction. A main effect of bias (step direction) was found in AP stepping direction $(F(2,27)=61.915, p<0.001$, $\eta \mathrm{p}^{2}=0.821$ ), where lengthening steps where undershot by a larger extend than shortening steps. No interaction effect for condition, step or group are found.

\section{Narrowing and widening the support base}

In ML direction the absolute error was greater for all groups in the reactive condition $(3.4 \pm 0.4 \mathrm{~cm})$ than the planned $(2.3 \pm 0.2 \mathrm{~cm})\left(\mathrm{F}(1,28)=11.073, \mathrm{p}=0.002, \eta \mathrm{p}^{2}=0.283\right)$ (Figure $\left.2 \mathrm{~B}\right)$. No interaction effects of step direction an group were found, but a main effect of step direction was found $(F(2,27)=15.912, p<0.001, \eta p 2=0.541)$. Preferred steps had significantly smaller error magnitudes than narrowing $(\mathrm{p}=0.001)$ and widening $(\mathrm{p}<0.001)$. Due to the covariation for SSWS SS $(4.5 \pm 0.6 \mathrm{~cm}, 1.9 \pm 0.2,5.1 \pm 0.7 \mathrm{~cm})$ did not have a different direction of error than YH $(2.4 \pm 0.6,1.5 \pm 0.2,1.7 \pm 0.7 \mathrm{~cm})$ and $\mathrm{OH}(3.3 \pm 0.6,1.2 \pm 0.3,3.7 \pm 0.8 \mathrm{~cm}))$ when lengthening, preferred and shortening respectively (Figure 3 ).

When investigation bias, a main effect of response time is found $\left(\mathrm{F}(1,28)=4.408, \mathrm{p}=0.045, \eta \mathrm{p}^{2}=0.136\right)$, showing steps in the reactive condition $(-0.4 \pm 0.1 \mathrm{~cm})$ are less undershot than in the planned condition $(-0.2 \pm 0.1 \mathrm{~cm})$. No main effect of group or step direction are found for the bias.

\section{Percentage of missed targets}

Overall fewer targets were missed in the planned (3.4 $1.3 \%)$ than reactive stepping condition $(4.7 \pm 1.3 \%, \mathrm{~F}(1$, $\left.29)=4.183, \mathrm{p}=0.05, \eta \mathrm{p}^{2}=0.126\right)$. SS $(9.8 \pm 2.1 \%)$ missed more targets than $\mathrm{YH}(1.0 \pm 2.1 \%, \mathrm{p}<0.001) \mathrm{OH}$ $(1.3 \pm 2.2 \%, \mathrm{p}=0.001)\left(\mathrm{F}(2,29)=11.419, \eta \mathrm{p}^{2}=0.441\right)$. However, no interaction effects were found for group and 
condition such that response time reduction was not seen to induce more missed targets for SS to a greater extent than $\mathrm{YH}$ and $\mathrm{OH}$ (figure 4).

\section{Discussion}

This is the first study to compare foot-placement accuracy during walking for planned and reactive targets in four adaptation directions (shortening, lengthening, narrowing and widening) between $\mathrm{YH}, \mathrm{OH}$ and SS. Overall reactive targets were missed more often than planned targets. Surprisingly, only a few between-group differences in foot-placement accuracy were found when stepping reactively and according to the direction of step adjustment. We consider these in detail to better understand difficulties SS face and any potential implications for rehabilitation.

When adjusting steps reactively more targets were missed (4.7\% vs 3.4\% for planned) by all groups as reported in previous studies $[15,16,24]$, however less pronounced than in the YH study by Hoogkamer, Potocanac [24]. Somewhat surprisingly, SS were not affected more by reduced response time than any other participant group. It is surprising that $\mathrm{YH}$ have such high failure rates, as they are known to be able to adjust foot-placement up until late stance of the targeting leg $[10,11]$. These differences could be explained by differences in paradigm complexity and task difficulty. The paradigms employed in this study varies from previously used dual-step paradigms [24], in which targets switch location with a set response time/distance, and from visual occlusion paradigms [10, 11], where vision gets occluded at phases of the task. In contrast in this study, targets only appeared at mid-stance of the contralateral leg. This limits the time to plan foot-placement but provides a context in which participants anticipate the continuous requirement to change their step. In this context, several plans for stepping trajectories may be planned in advance and those that are inappropriate inhibited [29].

In contrast to previous studies, SS showed little difference between reactive and planned foot-placement accuracy $[15,16]$. However, these previous studies report very different success rates to one another in SS. It could be argued that the timing of target presentation in the reactive condition of our study (at mid swing) was less challenging than the obstacle avoidance studies. In addition the stroke severity was greater in the previous studies, who included individuals with foot drop[16], with lower average walking speeds [15]. The small increase in failure rate in reactive stepping, in the recent study, may be due to differences in foot-placement planning between SS and healthy adults. Healthy adults are known to plan foot-placements about two steps ahead, switching gaze to the next target only when they have finished foot-placement $[10,11]$. When the terrain is cluttered, this gaze shift happens sooner. In contrast, populations with a high falls risk shift their gaze to the next foot-placement before the foot has landed on the target [30]. They do this even when the terrain is not cluttered resulting in reduced foot-placement accuracy. If SS indeed use this gaze strategy, you would expect a greater error and missed targets in all stepping conditions. The limited difference in error between reactive and planned foot-placement adjustments in SS could indicate a "cluttered terrain" gaze strategy even during planned stepping, which affords only a short time to plan foot-placement even when longer time is available (planned stepping). This could account for diminished difference between the planned and reactive condition (and explain the overall higher number of missed steps by SS). Future research could measure gaze strategies during gait adaptation paradigms in SS. 
Our evidence for this 'cluttered terrain strategy' is supported by the fact that SS and OH start 'falling behind the targets in this ongoing target stepping paradigm. This is seen in previous work assessing the effect of support for balance on foot placement accuracy[19]. In previous target stepping literature it has been shown YH participants overshoot shortening and undershoot lengthening targets [19, 24]. In this study SS, and to some extend $\mathrm{OH}$, seem to fall behind the ongoing target stepping paradigm (see fig 2A AP ABS error), their shortening steps are even undershot. When preferred foot-placement error would be subtracted from the adaptation foot-placement errors our results would be in agreement with Hoogkamer et al.[24], and show OH and SS would overshoot shortening and undershoot lengthening steps. However this would negate how SS fall behind or follow the steps, as previously seen in audio cueing studies[31] while YH anticipate the audio cues with their foot fall [31-33].

Somewhat surprisingly, our results showed that reactive foot-placement adjustments affected accuracy of foot-placement in ML direction differently than AP direction. Foot-placement errors in ML direction were increased during reactive stepping. Adjusting foot-placement in the ML direction may require lateral redirection of the forward momentum of the body, and with that ML adjustments require more active stabilization of the hip muscles[34]. Adjustments to steps in the ML direction may therefore, be more challenging than prolonging or terminating the already ongoing AP body momentum as ML adjustments have been shown to. This explanation is in line with proposals of Bancroft and Day [22] that magnitude and direction of the body momentum is specifically planned for the position of foot-placement. However, our results demonstrating lengthening steps have largest footplacement errors are in contradiction with both previous studies $[17,24]$ showing that base of support limiting steps were more challenging than enlarging steps.

We did not observe a difference in foot-placement error between the paretic and non-paretic leg in reactive stepping in SS. This could be explained in one of two ways: 1) stepping for both legs is affected to a similar extent by different impairments; impaired motor control of the paretic limb in aiming and impaired strength/balance during paretic stance, which affects aiming of the non-paretic limb, or 2) the stroke affects the bilateral control of both legs in a similar way. Both theories have been argued in previous literature. Said et al.[35] found obstacle crossing during paretic stance more challenging to balance than when standing on the non-paretic leg. The authors conclude that SS are less stable during paretic stance. In addition, Nonnekes et al. [14] found delayed muscle responses in adaptations during paretic swing, which led to slower reactions to the sudden appearance of a target. Both these impairments directly affect the ability to aim with either leg. Bilateral control is proposed because planning and execution of a successful foot-placement adjustment requires adjustment to both the aiming and stance legs[23]. Moreover, because the paretic side may show the most obvious impairments, bilateral effects of stroke are often overlooked. For example, Debaere et al. [36] found evidence for coordination deficits on the non-paretic side. In the present study paretic and non-paretic aim are affected similarly in both the absolute error and endpoint bias. Taken together these results suggest bilateral effects of stroke. Foot-placement requires control of both the stance and aiming limbs in SS and account for the lack of difference in aiming ability between paretic and non-paretic limbs.

\section{Limitations}


The main limitation of this study lies in the use of continuous target stepping paradigms, which may have equalized the intended challenge of the reactive targets compared to planned targets. Instead, the fact that participants were walking in a context priming them to anticipate continuous demands to adjust steps (regardless of whether they could see the step in advance or not) allowed us to examine how SS react to cluttered and dynamic environments. SS undershot targets, suggesting adjustments to foot-placement in cluttered environments maybe controlled in a "reactive" or "online" mode. This may be in contrast to YH who appear to be able to plan ahead even within the context of needing to adapt most steps. Further, the use of visual cues to specify targets may have served to draw and maintain attention to the task and in so doing actually improved the foot-placement AP adjustments in the reactive condition for all participant groups. This indicates that future studies and indeed treatment should focus on the interplay of attention/cognition and gait adaptability.

\section{Conclusion}

The results of this work confirm that the likelihood of SS misplacing a step in cluttered terrain is large; as one out of ten steps is likely to be misplaced, and could possibly lead to a fall. However, in contrast to healthy individuals, SS have overall worse foot-placement accuracy when adjusting steps in either planned or reactive conditions. The fact that $\mathrm{YH}$ and $\mathrm{OH}$ showed an increase in missed targets in the reactive condition compared to the planned condition while SS showed no differences in planned and reactive conditions, indicates a need for future studies to determine if SS may be responding to the need to adapt steps with the same control strategy regardless of whether the foot-fall target can be seen in advance or not. Further investigation is needed to understand footplacement control strategies used by SS and the role of attention/planning in gait adaptability in order to inform rehabilitation approaches.

Table 1: Participant demographics and clinical characteristics represented in mean \pm SD unless specified differently.

\begin{tabular}{|r|c|c|c|}
\hline & Young adults & Older adults & Stroke Survivors \\
\hline$N(F)$ & $11(7)$ & $10(3)$ & $11(2)$ \\
\hline Age (years) & $29.5 \pm 6.2$ & $63.8 \pm 7.9$ & $66.7 \pm 9.4$ \\
SSWS $(\mathrm{m} / \mathrm{s})$ & $1.09 \pm 0.18$ & $0.88 \pm 0.19$ & $0.54 \pm 0.21$ \\
Trial making test $A(s)$ & $24 \pm 12$ & $31 \pm 14$ & $56 \pm 36$ \\
Falls in the last 12 months & $50 \pm 28$ & $69 \pm 42$ & $406 \pm 919$ \\
No fall $(\mathrm{N})$ & - & $27.4 \pm 1.9$ & $26.6 \pm 2.9$ \\
1 fall & - & & 8 \\
$1<$ fall & - & 1 & 1 \\
\hline
\end{tabular}




\begin{tabular}{|r|c|c|c|}
\hline Apples test & - & - & $46.4 \pm 10.1$ \\
\hline Time since stroke (months) & - & - & $87.5 \pm 134.3$ \\
\hline Paretic (Right) & - & - & 5 \\
\hline Berg balance & - & - & $50.4 \pm 5.4$ \\
\hline Fugl-Meyer (lower limb) & - & - & $26 \pm 5.6$ \\
\hline Dynamic gait index & - & - & $24.8 \pm 4.6$ \\
\hline 10m-walking speed (s) & - & - & $12.9 \pm 4.3$ \\
\hline Timed up and go (s) & - & & $16.7 \pm 5.9$ \\
\hline
\end{tabular}

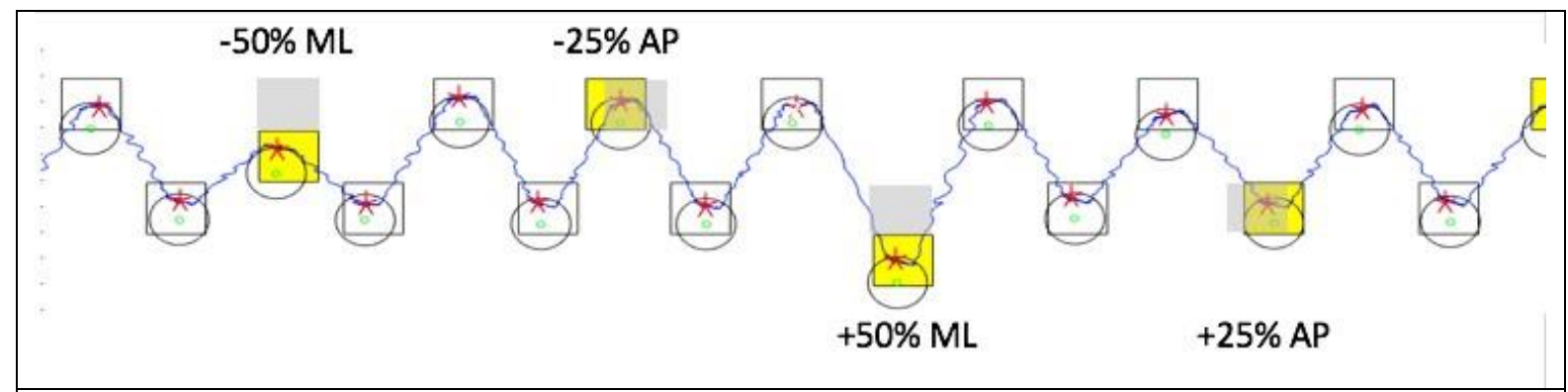

Figure 1: A schematic representation of the target positioning on the treadmill, white squares are preferred target positions, yellow targets represent the targets requiring adaptations to foot-placement (short/long, wide/narrow) with grey shadow of where the target would have been for preferred foot location. The Blue line represents the centre of pressure trajectory of one participant with the red asterisk representing centre of pressure at midstance. The green circle represents the centre of foot at time of midstance with the representation of the foot (larger circle around the centre of foot). AP, adjustments in anterior/posterior direction; $M L$, adjustments in medio-lateral direction. 


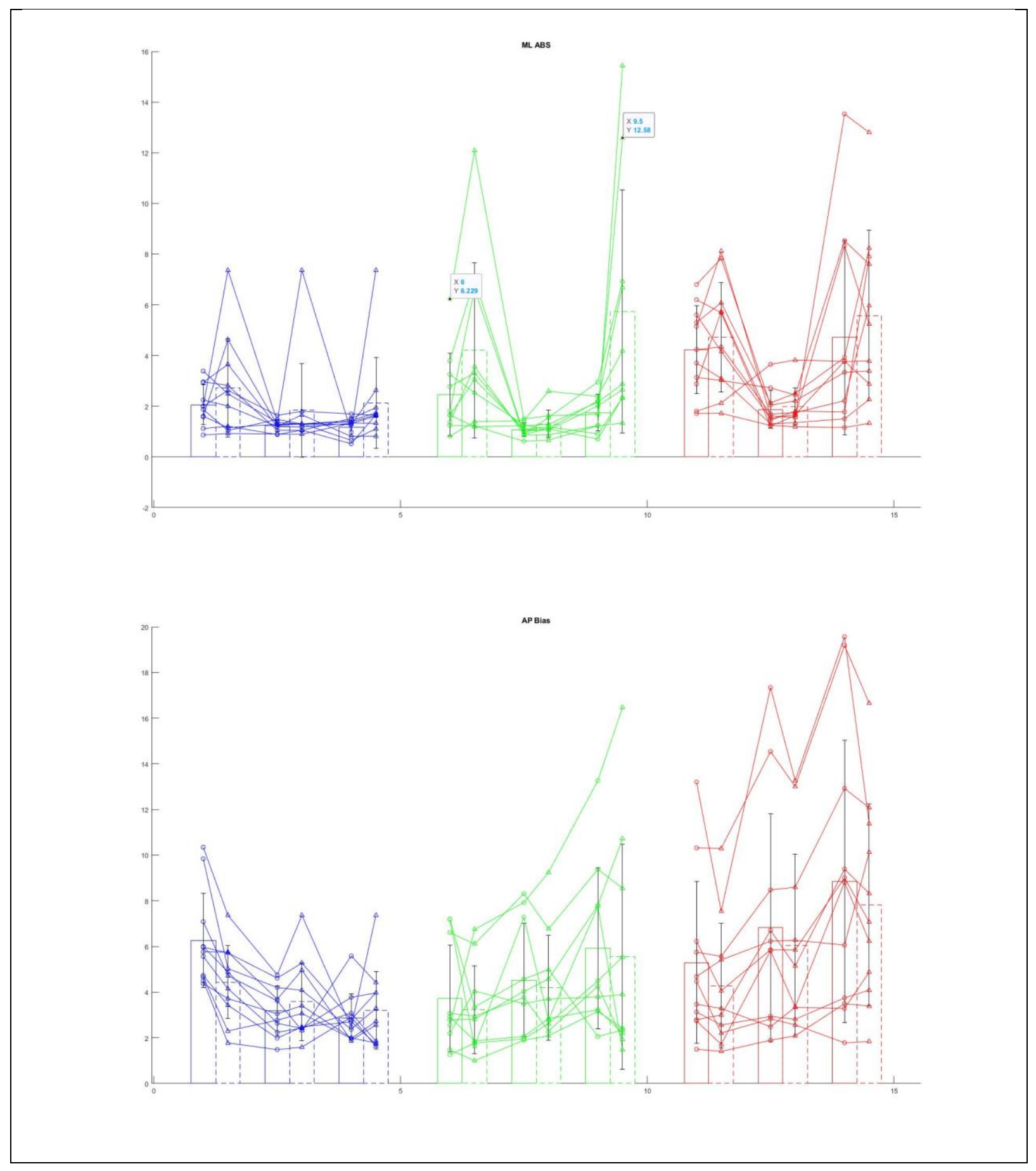




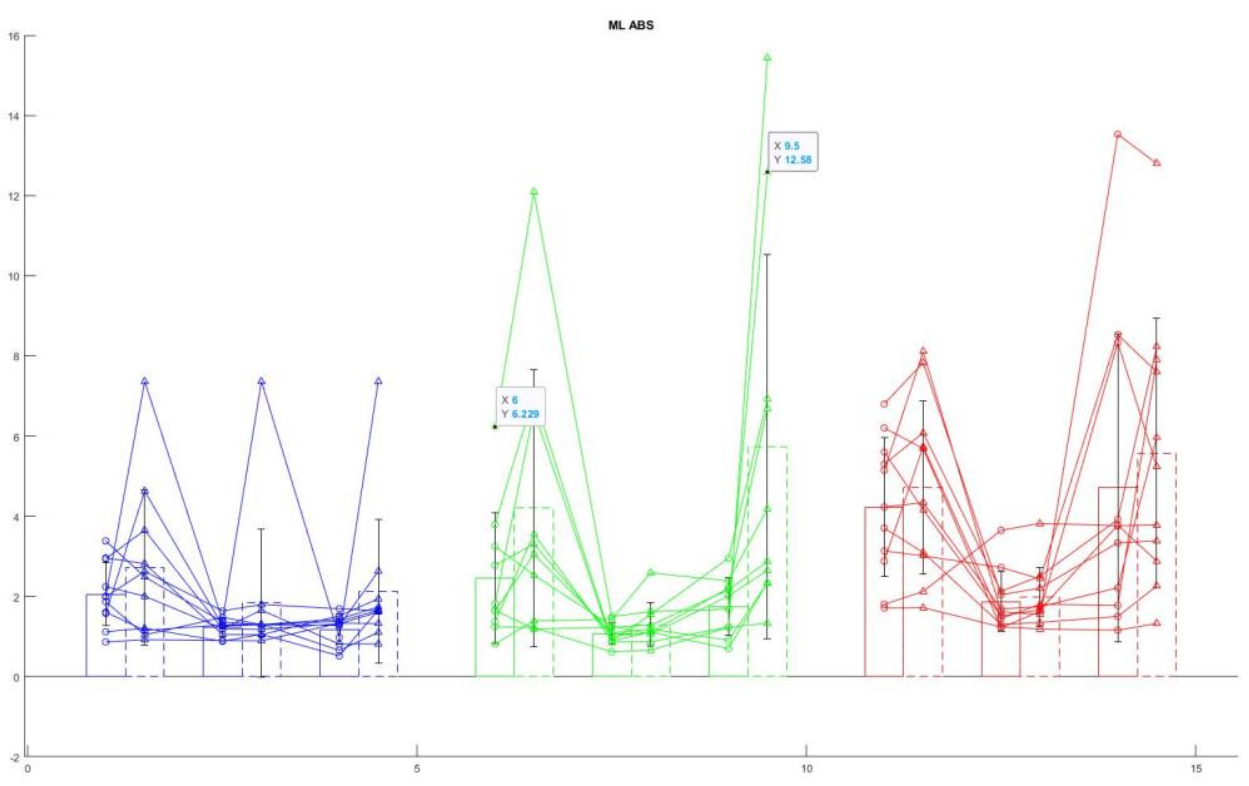

Figure 2: Bars represent the mean A) antero-posterior, and B) medio-lateral absolute error of foot-placement for young healthy adults $(\mathrm{YH})$ in blue, older healthy $(\mathrm{OH})$ in yellow and stroke survivors (SS) in red, for each type both planned (solid) and reactive (dashed) conditions. Error bars represent standard deviation. 


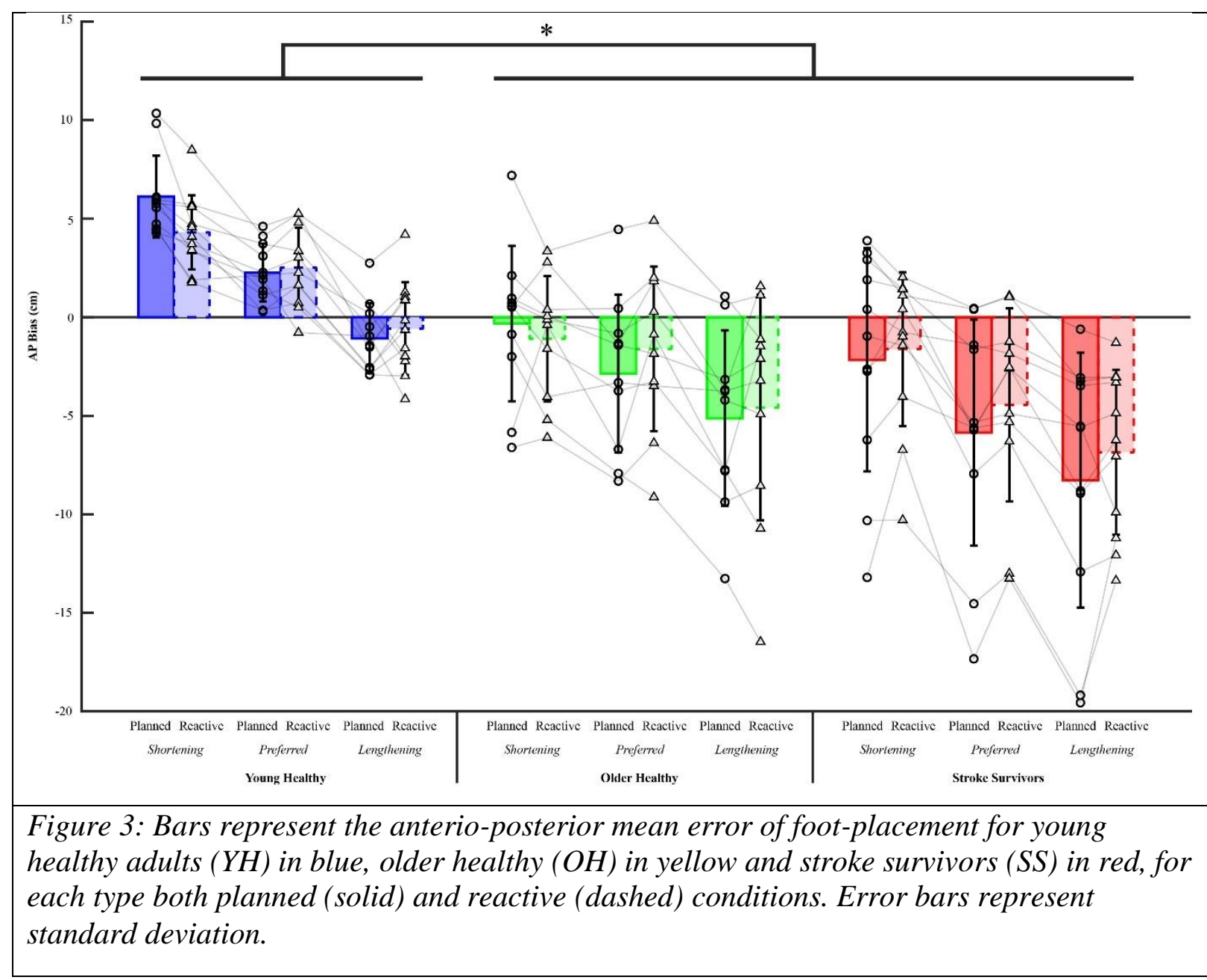




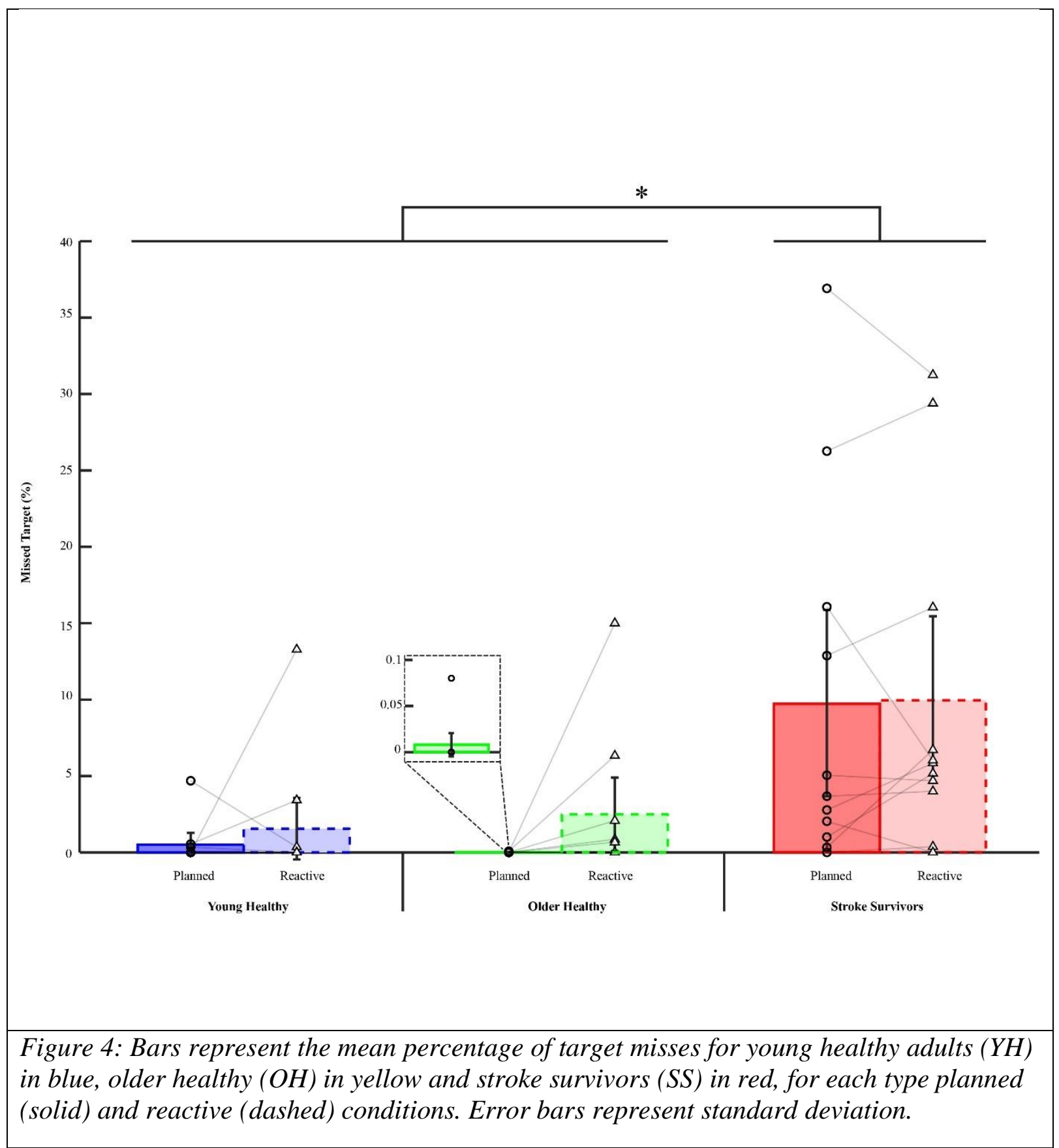

1. Hyndman, D., A. Ashburn, and E. Stack, Fall events among people with stroke living in the community: circumstances of falls and characteristics of fallers, in Arch Phys Med Rehabil. 2002. p. $165-170$.

2. Lamb, S.E., et al., Risk factors for falling in home-dwelling older women with stroke: the Women's Health and Aging Study, in Stroke. 2003: Interdisciplinary Research Centre in Health, School of Health and Social Sciences, Coventry University/Warwick West Midlands Primary Care Network, Coventry, UK. s.lamb@coventry.ac.uk. p. 494-501. 
3. Forster, A. and J. Young, Incidence and consequences of falls due to stroke: a systematic inquiry. BMJ, 1995. 311(6997): p. 83-6.

4. Jorgensen, L., T. Engstad, and B.K. Jacobsen, Higher incidence of falls in long-term stroke survivors than in population controls: depressive symptoms predict falls after stroke. Stroke, 2002. 33(2): p. 542-7.

5. Lord, S.E., et al., Community ambulation after stroke: how important and obtainable is it and what measures appear predictive?, in Archives of Physical Medicine and Rehabilitation. 2004. p. 234-239.

6. Berg, W.P., et al., Circumstances and consequences of falls in independent community-dwelling older adults. Age Ageing, 1997. 26(4): p. 261-268.

7. Blennerhassett, J.M., et al., Changes in balance and walking from stroke rehabilitation to the community: a follow-up observational study. Arch Phys Med Rehabil, 2012. 93(10): p. 1782-7.

8. Geerse, D.J., B.H. Coolen, and M. Roerdink, Walking-adaptability assessments with the Interactive Walkway: Between-systems agreement and sensitivity to task and subject variations, in Gait and Posture. 2017.

9. Hollands, K.L.L., et al., A novel and simple test of gait adaptability predicts gold standard measures of functional mobility in stroke survivors, in Gait and Posture. 2016. p. 170-175.

10. Hollands, M.A. and D.E. Marple-Horvat, Coordination of eye and leg movements during visually guided stepping, in J Mot Behav. 2001: Neural Control Laboratory, Department of Kinesiology, University of Waterloo, Waterloo, Ontario N2L 3G1, Canada. hollands@nclab.uwaterloo.ca. p. 205-216.

11. Matthis, J.S. and B.R. Fajen, Visual control of foot placement when walking over complex terrain, in J Exp Psychol Hum Percept Perform. 2014: Department of Cognitive Science, Rensselaer Polytechnic Institute. p. 106-115.

12. Young, W.R. and M.A. Hollands, Evidence for age-related decline in visuomotor function and reactive stepping adjustments, in Gait Posture. 2012: School of Psychology, Queen's University Belfast, Belfast, BT9 5BN, UK. w.young@qub.ac.uk. p. 477-481.

13. Peper, C.L., M.J. de Dreu, and M. Roerdink, Attuning one's steps to visual targets reduces comfortable walking speed in both young and older adults, in Gait Posture. 2015: MOVE Research Institute Amsterdam, Faculty of Human Movement Sciences, VU University Amsterdam, Amsterdam, The Netherlands. Electronic address: I.peper@vu.nl. MOVE Research Institute Amsterdam, Faculty of Human Movement Sciences, VU University Amsterdam,. p. 830834.

14. Nonnekes, J.H., et al., Deficits underlying impaired visually triggered step adjustments in mildly affected stroke patients, in Neurorehabil Neural Repair. 2010: UCL Institute of Neurology, London, United Kingdom, Radboud University, Nijmegen, The Netherlands. p. 393-400.

15. Den Otter, A.R., et al., Step characteristics during obstacle avoidance in hemiplegic stroke. Experimental Brain Research, 2005. 161(2): p. 180-192.

16. van Swigchem, R., et al., Deficits in motor response to avoid sudden obstacles during gait in functional walkers poststroke. Neurorehabil Neural Repair, 2013. 27(3): p. 230-9.

17. Moraes, R., F. Allard, and A.E. Patla, Validating determinants for an alternate foot placement selection algorithm during human locomotion in cluttered terrain. J Neurophysiol, 2007. 98(4): p. 1928-1940.

18. van Swigchem, R., et al., The capacity to restore steady gait after a step modification is reduced in people with poststroke foot drop using an ankle-foot orthosis, in Phys Ther. 2014: R. van Swigchem, PT, PhD, Department of Rehabilitation, Donders Centre for Neuroscience, Radboud University Medical Centre, Nijmegen, the Netherlands. p. 654-663. 
19. van der Veen, S.M., U. Hammerbeck, and K.L. Hollands, How accuracy of foot-placement is affected by the size of the base of support and crutch support in stroke survivors and healthy adults. Gait Posture, 2020. 76: p. 224-230.

20. van der Veen, S.M., et al., Validation of gait event detection by centre of pressure during target stepping in healthy and paretic gait, in Journal of Biomechanics. 2018, Elsevier.

21. Roerdink, M., et al., Online gait event detection using a large force platform embedded in a treadmill. J Biomech, 2008. 41(12): p. 2628-2632.

22. Bancroft, M.J. and B.L. Day, The Throw-and-Catch Model of Human Gait: Evidence from Coupling of Pre-Step Postural Activity and Step Location, in Front Hum Neurosci. 2016: Sobell Department of Motor Neuroscience and Movement Disorders, UCL Institute of Neurology, University College London London, UK. p. 635.

23. Reynolds, R.F. and B.L. Day, Visual guidance of the human foot during a step. J Physiol, 2005. 569(Pt 2): p. 677-84.

24. Hoogkamer, W., Z. Potocanac, and J. Duysens, Quick foot placement adjustments during gait: direction matters. Exp Brain Res, 2015.

25. Schmid, A., et al., Improvements in speed-based gait classifications are meaningful, in Stroke. 2007: Department of Occupational Therapy, Indiana University School of Health and Rehabilitation Sciences, and the Richard L. Roudebush VA Medical Center, Indianapolis, IN 46202-5119, USA. araschmi@iupui.edu. p. 2096-2100.

26. Brouwer, B., K. Parvataneni, and S.J. Olney, A comparison of gait biomechanics and metabolic requirements of overground and treadmill walking in people with stroke. Clin Biomech (Bristol, Avon), 2009. 24(9): p. 729-34.

27. Berg, K., et al., Measuring balance in the elderly-development of an instrument. Phys Ther, 1988. 68(5): p. 811.

28. Shumway-Cook, A., S. Brauer, and M. Woollacott, Predicting the Probability for Falls in Community-Dwelling Older Adults Using the Timed Up \& Go Test, in Physical Therapy. 2000.

29. Shumway-Cook, A., et al., Predicting the Probability for Falls in Community-Dwelling Older Adults. 1997.

30. Chapman, G.J. and M.A. Hollands, Evidence that older adult fallers prioritise the planning of future stepping actions over the accurate execution of ongoing steps during complex locomotor tasks, in Gait Posture. 2007: Human Movement Laboratory, School of Sport and Exercise Sciences, University of Birmingham, Edgbaston, Birmingham B15 2TT, United Kingdom. p. 59-67.

31. Roerdink, M., et al., Rhythm perturbations in acoustically paced treadmill walking after stroke, in Neurorehabil Neural Repair. 2009: Research Institute MOVE, Faculty of Human Movement Sciences, VU University, Department of Rehabiliation Medicine, VU University Medical Centre, Amsterdam, the Netherlands. m.roerdink@fbw.vu.nl.p. 668-678.

32. Bank, P.J., M. Roerdink, and C.E. Peper, Comparing the efficacy of metronome beeps and stepping stones to adjust gait: steps to follow! Exp Brain Res, 2011. 209(2): p. 159-69.

33. Roerdink, M., et al., Walking to the beat of different drums: practical implications for the use of acoustic rhythms in gait rehabilitation. Gait Posture, 2011. 33(4): p. 690-4.

34. Rankin, B.L., S.K. Buffo, and J.C. Dean, A neuromechanical strategy for mediolateral foot placement in walking humans, in Journal of Neurophysiology. 2014: Dean, Jc 77 President St,MSC700, Charleston, SC 29425 USA 77 President St,MSC700, Charleston, SC 29425 USA Med Univ S Carolina, Coll HIth Profess, Div Phys Therapy, Charleston, SC 29425 USA Ralph H Johnson Dept Vet Affairs Med Ctr, Charleston, SC USA. p. 374-383.

35. Said, C.M., et al., Balance during obstacle crossing following stroke, in Gait Posture. 2008:

Heidelberg Repatriation Hospital, Heidelberg West, 3081, Victoria, Australia.

Cathy.Said@austin.org.au.p. 23-30. 
36. Debaere, F., et al., Brain areas involved in interlimb coordination: a distributed network, in Neuroimage. 2001: Motor Control Laboratory, Department of Radiology, K.U. Leuven, Tervuurse Vest 101, Heverlee, 3001, Belgium. p. 947-958. 